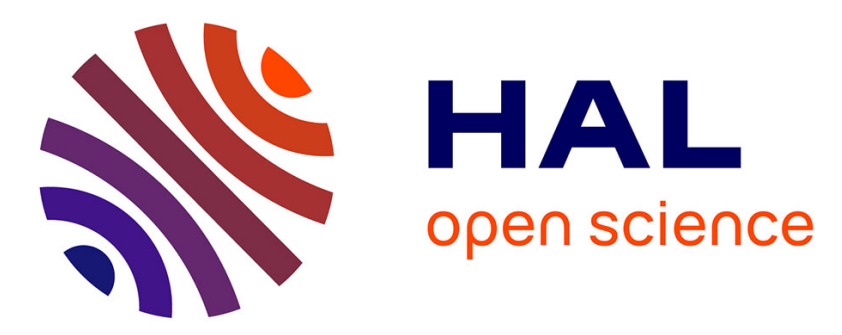

\title{
Power allocation in Uplink Multiband Satellite System with Nonlinearity-Aware Receiver
}

\author{
Arthur Louchart, Philippe Ciblat, Charly Poulliat
}

\section{To cite this version:}

Arthur Louchart, Philippe Ciblat, Charly Poulliat. Power allocation in Uplink Multiband Satellite System with Nonlinearity-Aware Receiver. IEEE Workship on Signal Processing Advances on Wireless Communications (SPAWC), 2021, Lucca (virtual), Italy. hal-03291071

\section{HAL Id: hal-03291071 \\ https://hal.science/hal-03291071}

Submitted on 19 Jul 2021

HAL is a multi-disciplinary open access archive for the deposit and dissemination of scientific research documents, whether they are published or not. The documents may come from teaching and research institutions in France or abroad, or from public or private research centers.
L'archive ouverte pluridisciplinaire HAL, est destinée au dépôt et à la diffusion de documents scientifiques de niveau recherche, publiés ou non, émanant des établissements d'enseignement et de recherche français ou étrangers, des laboratoires publics ou privés. 


\section{Power allocation in Uplink Multiband Satellite System with Nonlinearity-Aware Receiver}

\author{
Arthur Louchart \\ Telecom Paris \\ Institut Polytechnique de Paris \\ Palaiseau, France \\ arthur.louchart@telecom-paris.fr
}

\author{
Philippe Ciblat \\ Telecom Paris \\ Institut Polytechnique de Paris \\ Palaiseau, France \\ philippe.ciblat@telecom-paris.fr
}

\author{
Charly Poulliat \\ INP-ENSEEIHT \\ Toulouse, France \\ charly.poulliat@enseeiht.fr
}

\begin{abstract}
In this paper, we address power allocation for an uplink multiband single-beam satellite system taking into account on-board nonlinearities. These nonlinearities are generated by the high power amplifier. Based on the closed-form expression of the capacity associated with the optimal receiver exploiting the structure of the nonlinear effects, three power allocations are studied: maximization of the sum-rate, maximization of the minimum of the rates, and minimization of the sum of the powers. The related optimization problems boil down nonconvex problems that can be cast and solved by using signomial programming. We propose practical and scalable algorithms for fixing these power allocation problems.
\end{abstract}

Index Terms-Power allocation, nonlinear regime, signomial programming, HPA, capacity

\section{INTRODUCTION}

Communication networks need to carry more and more data with new user cases. The hybridization of cellular and satellite networks allows to answer the exponential increase of data traffic. Indeed, the satellite system plays the role of a relay between two points of a terrestrial system. The satellite uplink/return link is studied in this paper, where terrestrial users transmit data to the satellite, which then sends it to a terrestrial gateway.

In order to respond to the increase in data rates, a new frequency range around the Ka-band is considered. In this context, some studies have proposed resource allocation algorithms to maximize the system capacity [1], [2]. However, these studies assume that the satellite high-power amplifier (HPA) operates in the linear regime.

When nonlinear regime of the HPA is taken into account, system capacity expressions have been provided in [3]. Actually the authors exhibited two different expressions according to the way the nonlinear interference has been treated by the receiver. The first way is called nonlinearityagnostic since the nonlinear interference is seen as an extra noise. The second way is called nonlinearity-aware since the nonlinear interference is seen as an extra signal carrying useful information. In both cases, significant gains have been observed if optimal power allocations are done. Nevertheless, the optimal power allocations relied on an exhaustive search,

This work has been funded by ANR through the SIERRA FrenchLuxembourgian grant. which is not scalable with an increasing number of users. Practical and scalable power allocations have been proposed in [4] for the case of nonlinearity-agnostic receivers. In this paper, we aim to propose power allocation algorithms for the nonlinearity-aware receivers. We will show that the capacity expression involves a posynomial over signomial ratio, leading to Signomial Programing (SP) optimization problem. To the best of our knowledge, this type of problem has never been used in the field of wireless communications.

In [4], [5], the presence of the linear and nonlinear interference respectively involves a ratio of posynomials in the capacity and is thus solved using the same methodology, i.e., Successive Convex Approximation (SCA) followed by Geometric Programing (GP). Here, this approach is not suitable. As initially seen by [6], we suggest moving the ratio of signomials into the constraint set leads to a ratio of posynomials in the constraint set. Then similarly to [4], [5], SCA procedure followed by GP can be used to solve the considered optimization problems. We show that this approach leads to practical and scalable power allocation algorithms.

The rest of the paper is organized as follows: in Section II, we introduce the system model. In Section III, we present and solve the three different power allocation problems. Numerical illustrations are given in Section IV. Section V is devoted to concluding remarks and perspectives.

\section{SYSTEM MODEL}

A single-beam multiband satellite communication system is considered, where $K$ terrestrial users transmit data towards the satellite. In order to avoid interference on the uplink, the users share orthogonal subbands (FDMA) [7]. We assume that the assignment between users and subbands has been already done, so that there are as many active users as subbands.

Each user $k \in\{1, \cdots, K\}$ transmits its own sequence of independent and identically-distributed symbol $\left\{a_{k, n}\right\}_{n \in \mathbb{Z}}$ to the satellite. We denote by $P_{k}=\mathbb{E}\left[\left|a_{k, n}\right|^{2}\right]$ the transmit power of user $k$. The link between user $k$ and the satellite is characterized by the channel gain $G_{k}$ and is computed according to the user location [7]. Inside the satellite, the HPA amplifies the sum of received signal and produces nonlinear effects. The downlink, from the satellite to the gateway, is 
assumed to be perfect. Therefore, the received samples at the gateway, denoted by $z_{k, n}$ for user $k$, are written as below [3]:

$$
z_{k, n}=z_{k, n}^{\mathrm{L}}+z_{k, n}^{\mathrm{NL}}+w_{k, n}
$$

with

$$
\begin{aligned}
z_{k, n}^{\mathrm{L}} & =\omega_{1} \sqrt{G_{k}} a_{k, n} \\
z_{k, n}^{\mathrm{NL}} & =\omega_{3} \sum_{k_{1}, k_{2}, k_{3}=1}^{K} \sum_{n_{1}, n_{2}, n_{3} \in \mathbb{Z}} a_{k_{1}, n-n_{1}} a_{k_{2}, n-n_{2}} a_{k_{3}, n-n_{3}}^{*} \\
& \times \sqrt{G_{k_{1}} G_{k_{2}} G_{k_{3}}} e^{2 i \pi\left(k_{1}+k_{2}-k_{3}-k\right) \Delta B n T_{s}} \\
& \times h_{3}\left(n_{1} T_{s}, n_{2} T_{s}, n_{3} T_{s}, k_{1}+k_{2}-k_{3}-k\right)
\end{aligned}
$$

where $\Delta B$ is the subband size and $w_{k, n}$ is the additive white zero-mean Gaussian noise (AWGN) of variance $\mathcal{P}_{\mathrm{W}}=$ $\mathbb{E}\left[\left|w_{k, n}\right|^{2}\right]$. The HPA is characterized by $\omega_{1}$ and $\omega_{3}$, which are positive coefficients. The Volterra kernel $h_{3}\left(t_{1}, t_{2}, t_{3}, m\right)$ is written as follows:

$$
h_{3}\left(t_{1}, t_{2}, t_{3}, m\right)=\int_{\mathbb{R}} \prod_{\ell=1}^{3} p_{T}\left(t_{\ell}-\tau\right) p_{R}(\tau) e^{-i 2 \pi m \Delta B \tau} d \tau
$$

where $p_{T}(t)$ and $p_{R}(t)$ are the shaping filter and the corresponding matched filter. Note that $p_{T}(t)$ is a square-root Nyquist filter.

As the nonlinear interference $\left(z_{k, n}^{\mathrm{NL}}\right)$ is correlated to the useful signal $\left(z_{k, n}^{\mathrm{L}}\right)$, this nonlinear interference can be seen as an additional information. In that case, according to [3], the expression of the capacity for user $k$ is

$$
C(k)=\log _{2}(1+S(k)),
$$

with

$$
S(k)=\frac{\mathcal{P}_{\mathrm{L}}^{2}(k)+2 \mathcal{P}_{\mathrm{L}}(k) \mathcal{P}_{\mathrm{LNL}}(k)+\mathcal{P}_{\mathrm{LNL}}(k)^{2}}{\mathcal{P}_{\mathrm{L}}(k) \mathcal{P}_{\mathrm{W}}+\mathcal{P}_{\mathrm{L}}(k) \mathcal{P}_{\mathrm{NL}}(k)-\mathcal{P}_{\mathrm{LNL}}(k)^{2}} .
$$

The term $\mathcal{P}_{\mathrm{L}}(k):=\mathbb{E}\left[\left|z_{k, n}^{\mathrm{L}}\right|^{2}\right]$ is given by

$$
\mathcal{P}_{\mathrm{L}}(k)=\omega_{1}^{2} G_{k} P_{k} \text {. }
$$

The term $\mathcal{P}_{\mathrm{LNL}}(k):=\mathbb{E}\left[z_{k, n}^{\mathrm{L}}\left(z_{k, n}^{\mathrm{NL}}\right)^{*}\right]$ is given by

$$
\mathcal{P}_{\mathrm{LNL}}(k)=2 \omega_{1} \omega_{3} \psi G_{k} P_{k} \sum_{k^{\prime}=1}^{K} G_{k^{\prime}} P_{k^{\prime}} .
$$

The term $\mathcal{P}_{\mathrm{NL}}(k):=\mathbb{E}\left[\left|z_{k, n}^{\mathrm{NL}}\right|^{2}\right]$ is given by

$$
\begin{aligned}
\mathcal{P}_{\mathrm{NL}}(k) & =4 \omega_{3}^{2} \theta^{(1)} G_{k} P_{k} \sum_{k^{\prime}, k^{\prime \prime}=1}^{K} G_{k^{\prime}} G_{k^{\prime \prime}} P_{k^{\prime}} P_{k^{\prime \prime}} \\
& +2 \omega_{3}^{2} \theta^{(2)} \sum_{\substack{k_{1}, k_{2}, k_{3}=1 \\
k=k_{1}+k_{2}-k_{3}}}^{K} G_{k_{1}} G_{k_{2}} G_{k_{3}} P_{k_{1}} P_{k_{2}} P_{k_{3}} \\
& +4 \omega_{3}^{2} \phi^{(1)}\left(\tilde{\delta}_{k, 1} G_{k-1} P_{k-1}+\tilde{\delta}_{k, K} G_{k+1} P_{k+1}\right) \\
& \times \sum_{k^{\prime}, k^{\prime \prime}=1}^{K} G_{k^{\prime}} G_{k^{\prime \prime}} P_{k^{\prime}} P_{k^{\prime \prime}} \\
& +2 \omega_{3}^{2} \phi^{(2)} \sum_{\substack{k_{1}, k_{2}, k_{3}=1 \\
k=k_{1}+k_{2}-k_{3} \pm 1}}^{K} G_{k_{1}} G_{k_{2}} G_{k_{3}} P_{k_{1}} P_{k_{2}} P_{k_{3}},
\end{aligned}
$$

where $\tilde{\delta}_{k, k^{\prime}}=1-\delta_{k, k^{\prime}}$ with $\delta_{k, k^{\prime}}$ being the Kronecker index. The coefficients $\theta^{(i)}, \phi^{(i)}$ and $\psi$ are positive, and depend on the Volterra kernel [3].

In next section, we explore different allocation strategies for the transmit power vector $\mathbf{P}=\left[P_{1}, \cdots, P_{K}\right]$, where the data rate for user $k$ is evaluated with (1).

\section{POWER ALLOCATION STRATEGIES FOR NONLINEAR IMPAIRMENTS AWARE RECEIVERS}

We henceforth focus on three different power allocation problems. We propose a reformulation for each problem from which an efficient optimization method can be exhibited.

For all investigated problems, the same power mask constraint is assumed on the transmit power for any user $k$, i.e.

$$
0 \leq P_{k} \leq P_{\max } \quad \forall k=1, \ldots, K,
$$

where $P_{\max }$ represents the maximum transmit power.

We bring out some mathematical properties for $\mathcal{P}_{\mathrm{L}}(k)$, $\mathcal{P}_{\mathrm{LNL}}(k), \mathcal{P}_{\mathrm{NL}}(k)$ and the resulting capacity $C(k)$. To this aim, let us consider the three definitions below.

Definition 1: A monomial function takes the following form:

$$
m\left(P_{1}, \ldots, P_{K}\right)=c P_{1}^{b_{1}} \ldots P_{K}^{b_{K}}
$$

with $c \in \mathbb{R}^{+}$and $b_{k} \in \mathbb{R}$.

Definition 2: A posynomial function takes the following form:

$$
p\left(P_{1}, \ldots, P_{K}\right)=\sum_{n=1}^{N} m_{n}\left(P_{1}, \ldots, P_{K}\right)
$$

where $\left\{m_{n}\right\}_{n=1, \cdots, N}$ are monomial functions.

Definition 3: A signomial function takes the following form:

$$
s\left(P_{1}, \ldots, P_{K}\right)=p\left(P_{1}, \ldots, P_{K}\right)-q\left(P_{1}, \ldots, P_{K}\right)
$$

where $p$ and $q$ are posynomial functions.

Consequently, (3) is a monomial function and (4)-(5) are posynomial with respect to $\mathbf{P}$. We also notice that the denominator of (2) is a signomial function with respect to $\mathbf{P}$. We remind that GP refers to an optimization problem in which the objective function and inequality constraints are posynomial and it can be solved using convex programing after a change of variable [8].

\section{A. Sum-rate maximization}

We consider the system's sum-rate maximization. The related problem writes as follows:

Problem 1:

$$
\max _{\mathbf{P}} \sum_{k=1}^{K} \log _{2}(1+S(k)) \text { s.t. } \quad(\mathrm{C} 1) .
$$

As the logarithmic function is monotonically increasing, Problem 1 has the following equivalent formulation:

$$
\max _{\mathbf{P}} \prod_{k}(1+S(k)) \quad \text { s.t. } \quad(\mathrm{C} 1) .
$$

We notice that the objective function is a ratio of a posynomial over a signomial, leading to SP. As in [6], we introduce new 
variables $\mathbf{t}=\left[t_{1}, \cdots, t_{K}\right], t_{k} \geq 1$, in order to move the signomial functions into the constraint set. The problem is then written:

$$
\begin{aligned}
& \min _{\mathbf{P}, \mathbf{t}} \prod_{k}\left(t_{k}\right)^{-1} \text { s.t. }(\mathrm{C} 1) \\
& t_{k} \leq 1+S(k), \quad \forall k=1, \ldots, K
\end{aligned}
$$

A signomial constraint can always be transformed into a ratio of posynomial [5], [6]. Indeed, as the numerator and the denominator of $S(k)$ are positive [3], (C2) can be rewritten as

$$
\frac{t_{k}\left(\mathcal{P}_{\mathrm{NL}}(k)+\mathcal{P}_{\mathrm{W}}\right)}{D_{k}(\mathbf{P}, \mathbf{t})} \leq 1, \quad \forall k
$$

where $D_{k}(\mathbf{P}, \mathbf{t})$ is a posynomial function defined as

$$
\begin{aligned}
D_{k}(\mathbf{P}, \mathbf{t})=\mathcal{P}_{\mathrm{NL}}(k)+t_{k} & \mathcal{P}_{\mathrm{L}}^{-1}(k) \mathcal{P}_{\mathrm{LNL}}^{2}(k) \\
& +2 \mathcal{P}_{\mathrm{LNL}}(k)+\mathcal{P}_{\mathrm{L}}(k)+\mathcal{P}_{\mathrm{W}} .
\end{aligned}
$$

The resulting problem is still not in GP form, because $D_{k}(\mathbf{P}, \mathbf{t})$ is not a monomial function. As a consequence, we must deal with a nonconvex optimization problem. The Successive Convex Approximation (SCA) method is a common way to converge at a stationary point in nonconvex optimization problem, when the nonconvex constraints can be upper-bounded by convex functions. To work well, the upperbound, realized at a given point, must be close enough to the original function. The convexified problem is then solved to provide a new point. If the SCA conditions are satisfied, this procedure converges to a stationary point. The conditions on the convex upper-bound $\tilde{f}_{i}$ at iteration $i$ of the nonconvex function $f$ are: $i) f\left(\mathbf{x}_{i}\right)=\tilde{f}_{i}\left(\mathbf{x}_{i}\right)$, ii) $f(\mathbf{x}) \leq \tilde{f}_{i}(\mathbf{x}), \forall \mathbf{x}$, and iii) $\nabla f\left(\mathbf{x}_{i}\right)=\nabla \tilde{f}_{i}\left(\mathbf{x}_{i}\right)$ where $\mathbf{x}_{i}$ is the given point at iteration $i$, resulting from the solution of the previous iteration.

We therefore need to establish a tight upper-bound of our nonconvex constraint set, so that we can apply the SCA procedure. Following [6], [5], we use a monomial approximation of the denominator (6) that satisfies the SCA conditions. At iteration $i$ (during which the approximation is done around the point $\left.\left(\mathbf{P}_{i}, \mathbf{t}_{i}\right)\right)$, we solve the following problem, which leads to the next point $\left(\mathbf{P}_{i+1}, \mathbf{t}_{i+1}\right)$ :

$$
\begin{aligned}
&\left(P_{1}^{\prime}\right): \min _{\mathbf{P}, \mathbf{t}} \prod_{k}\left(t_{k}\right)^{-1} \text { s.t. }(\mathrm{C} 1) \\
& \qquad \frac{t_{k}\left(\mathcal{P}_{\mathrm{NL}}(k)+\mathcal{P}_{\mathrm{W}}\right)}{\widetilde{D}_{k, i}(\mathbf{P}, \mathbf{t})} \leq 1, \forall k
\end{aligned}
$$

where $\widetilde{D}_{k, i}(\mathbf{P}, \mathbf{t})$ is a monomial approximation of (6) at the point $\left(\mathbf{P}_{i}, \mathbf{t}_{i}\right)$. Problem $P_{1}^{\prime}$ is GP with respect to $(\mathbf{P}, \mathbf{t})$, and numerical algorithms can solve it efficiently [8]. In order the constraint functions (C3) to satisfy the SCA conditions for the original constraint functions (C2), we have

$$
\begin{aligned}
& \widetilde{D}_{k, i}(\mathbf{P}, \mathbf{t})=\prod_{k^{\prime}, k^{\prime \prime}=1}^{K}\left(\frac{4 \omega_{3}^{2} \theta^{(1)} G_{k} P_{k} G_{k^{\prime}} G_{k^{\prime \prime}} P_{k^{\prime}} P_{k^{\prime \prime}}}{\alpha_{k^{\prime}, k^{\prime \prime}}^{(1)}(k)}\right)^{\alpha_{k^{\prime}, k^{\prime \prime}}^{(1)}(k)} \\
& \times \prod_{\substack{k_{1}, k_{2}, k_{3}=1 \\
k=k_{1}+k_{2}-k_{3}}}^{K}\left(\frac{2 \omega_{3}^{2} \theta^{(2)} G_{k_{1}} G_{k_{2}} G_{k_{3}} P_{k_{1}} P_{k_{2}} P_{k_{3}}}{\alpha_{k_{1}, k_{2}, k_{3}}^{(2)}(k)}\right)^{\alpha_{k_{1}, k_{2}, k_{3}}^{(2)}(k)} \\
& \times \prod_{k^{\prime}, k^{\prime \prime}=1}^{K}\left(\frac{4 \omega_{3}^{2} \phi^{(1)} \tilde{\delta}_{k, 1} G_{k-1} P_{k-1} G_{k^{\prime}} G_{k^{\prime \prime}} P_{k^{\prime}} P_{k^{\prime \prime}}}{\alpha_{k^{\prime}, k^{\prime \prime}}^{(3)}(k)}\right)^{\alpha_{k^{\prime}, k^{\prime \prime}}^{(3)}(k)} \\
& \times \prod_{k^{\prime}, k^{\prime \prime}=1}^{K}\left(\frac{4 \omega_{3}^{2} \phi^{(1)} \tilde{\delta}_{k, K} G_{k+1} P_{k+1} G_{k^{\prime}} G_{k^{\prime \prime}} P_{k^{\prime}} P_{k^{\prime \prime}}}{\alpha_{k^{\prime}, k^{\prime \prime}}^{(4)}(k)}\right)^{\alpha_{k^{\prime}, k^{\prime \prime}}^{(4)}(k)} \\
& \times \prod_{\substack{k_{1}, k_{2}, k_{3}=1 \\
k=k_{1}+k_{2}-k_{3} \pm 1}}^{K}\left(\frac{2 \omega_{3}^{2} \phi^{(2)} G_{k_{1}} G_{k_{2}} G_{k_{3}} P_{k_{1}} P_{k_{2}} P_{k_{3}}}{\alpha_{k_{1}, k_{2}, k_{3}}^{(5)}}\right)^{\alpha_{k_{1}, k_{2}, k_{3}}^{(5)}} \\
& \times \prod_{k^{\prime}, k^{\prime \prime}=1}^{K}\left(\frac{4 \omega_{3}^{2} \psi^{2} t_{k} G_{k} P_{k} G_{k^{\prime}} P_{k^{\prime}} G_{k^{\prime \prime}} P_{k^{\prime \prime}}}{\beta_{k^{\prime}, k^{\prime \prime}}(k)}\right)^{\beta_{k^{\prime}, k^{\prime \prime}}(k)} \\
& \times \prod_{k^{\prime}=1}^{K}\left(\frac{4 \omega_{1} \omega_{3} \psi G_{k} P_{k} G_{k^{\prime}} P_{k^{\prime}}}{\gamma_{k^{\prime}}(k)}\right)^{\gamma_{k^{\prime}}(k)} \\
& \times\left(\frac{\omega_{1}^{2} G_{k} P_{k}}{\mu(k)}\right)^{\mu(k)}\left(\frac{\mathcal{P}_{\mathrm{W}}}{\eta(k)}\right)^{\eta(k)}
\end{aligned}
$$

with

$$
\begin{aligned}
& \alpha_{k^{\prime}, k^{\prime \prime}}^{(1)}(k)=\frac{4 \omega_{3}^{2} \theta^{(1)} G_{k} P_{k, i} G_{k^{\prime}} G_{k^{\prime \prime}} P_{k^{\prime}, i} P_{k^{\prime \prime}, i}}{D_{k}\left(\mathbf{P}_{i}, \mathbf{t}_{i}\right)} \\
& \alpha_{k_{1}, k_{2}, k_{3}}^{(2)}(k)=\frac{2 \omega_{3}^{2} \theta^{(2)} G_{k_{1}} G_{k_{2}} G_{k_{3}} P_{k_{1}, i} P_{k_{2}, i} P_{k_{3}, i}}{D_{k}\left(\mathbf{P}_{i}, \mathbf{t}_{i}\right)} \\
& \alpha_{k^{\prime}, k^{\prime \prime}}^{(3)}(k)=\frac{4 \omega_{3}^{2} \phi^{(1)} \tilde{\delta}_{k, 1} G_{k-1} P_{k-1, i} G_{k^{\prime}} G_{k^{\prime \prime}} P_{k^{\prime}, i} P_{k^{\prime \prime}, i}}{D_{k}\left(\mathbf{P}_{i}, \mathbf{t}_{i}\right)} \\
& \alpha_{k^{\prime}, k^{\prime \prime}}^{(4)}(k)=\frac{4 \omega_{3}^{2} \phi^{(1)} \tilde{\delta}_{k, K} G_{k+1} P_{k+1, i} G_{k^{\prime}} G_{k^{\prime \prime}} P_{k^{\prime}, i} P_{k^{\prime \prime}, i}}{D_{k}\left(\mathbf{P}_{i}, \mathbf{t}_{i}\right)} \\
& \alpha_{k_{1}, k_{2}, k_{3}}^{(5)}(k)=\frac{2 \omega_{3}^{2} \phi^{(2)} G_{k_{1}} G_{k_{2}} G_{k_{3}} P_{k_{1}, i} P_{k_{2}, i} P_{k_{3}, i}}{D_{k}\left(\mathbf{P}_{i}, \mathbf{t}_{i}\right)} \\
& \beta_{k^{\prime}}(k)=\frac{4 \omega_{3}^{2} \psi^{2} t_{k, i} G_{k} P_{k} G_{k^{\prime}} P_{k^{\prime}, i} G_{k^{\prime \prime}} P_{k^{\prime \prime}, i}}{D_{k}\left(\mathbf{P}_{i}, \mathbf{t}_{i}\right)} \\
& \gamma_{k^{\prime}}(k)=\frac{4 \omega_{1} \omega_{3} \psi G_{k} P_{k, i} G_{k^{\prime}} P_{k^{\prime}, i}}{D_{k}\left(\mathbf{P}_{i}, \mathbf{t}_{i}\right)}, \\
& \mu(k)=\frac{\omega_{1}^{2} G_{k} P_{k, i}}{D_{k}\left(\mathbf{P}_{i}, \mathbf{t}_{i}\right)}, \text { and } \eta(k)=\frac{\mathcal{P}_{\mathrm{W}}}{D_{k}\left(\mathbf{P}_{i}, \mathbf{t}_{i}\right)}
\end{aligned}
$$

After convergence of the SCA procedure [6], we obtain a local optimum point for the considered problem. It is interesting to note that the used approach is similar to SCA applied to Difference of Convex (DC), by applying the logarithm function to $(\mathrm{C} 2)$, exponential change of variables, and linear approximation of the second convex function in DC [9]. 


\section{B. Maximization of the minimum per-user data rate}

We study here the maximization of the minimum individual data rate. The corresponding problem states as follows:

Problem 2:

$$
\max _{\mathbf{P}} \min _{k} \log _{2}(1+S(k)) \quad \text { s.t. } \quad(\mathrm{C} 1)
$$

As the logarithmic function is monotonically increasing, Problem 2 has the following equivalent formulation:

$$
\max _{\mathbf{P}} \min _{k} \frac{\mathcal{P}_{\mathrm{L}}^{2}(k)+2 \mathcal{P}_{\mathrm{L}}(k) \mathcal{P}_{\mathrm{LNL}}(k)+\mathcal{P}_{\mathrm{LNL}}^{2}(k)}{\mathcal{P}_{\mathrm{L}}(k) \mathcal{P}_{\mathrm{NL}}(k)+\mathcal{P}_{\mathrm{L}}(k) \mathcal{P}_{\mathrm{W}}-\mathcal{P}_{\mathrm{LNL}}^{2}(k)} \quad \text { s.t. }
$$

where the objective function is a ratio of a posynomial over a signomial. As previously, the main idea is to move this ratio into the constraint set. By introducing the epigraph form as in [10], the following problem is obtained

$$
\begin{aligned}
\min _{\mathbf{P}, t} t^{-1} & \\
\text { s.t. } & (\mathrm{C} 1) \\
& \frac{\mathcal{P}_{\mathrm{L}}^{2}(k)+2 \mathcal{P}_{\mathrm{L}}(k) \mathcal{P}_{\mathrm{LNL}}(k)+\mathcal{P}_{\mathrm{LNL}}^{2}(k)}{\mathcal{P}_{\mathrm{L}}(k) \mathcal{P}_{\mathrm{NL}}(k)+\mathcal{P}_{\mathrm{L}}(k) \mathcal{P}_{\mathrm{W}}-\mathcal{P}_{\mathrm{LNL}}^{2}(k)} \geq t, \forall k
\end{aligned}
$$

As in Problem 1, we transform the last constraints into a ratio of posynomials given by

$$
\frac{t\left(\mathcal{P}_{\mathrm{NL}}(k)+\mathcal{P}_{\mathrm{W}}\right)}{(t+1) \mathcal{P}_{\mathrm{L}}^{-1}(k) \mathcal{P}_{\mathrm{LNL}}^{2}(k)+\mathcal{P}_{\mathrm{L}}(k)+2 \mathcal{P}_{\mathrm{LNL}}(k)} \leq 1, \quad \forall k .
$$

Finally, the management of the posynomial ratio is done in the same way as before, namely using the SCA procedure with a monomial approximation of the denominator.

\section{Sum-power minimization}

We now address the sum-power minimization under a peruser target data rate constraint. The problem writes as follows:

Problem 3:

$$
\begin{aligned}
\min _{\mathbf{P}} \sum_{k=1}^{K} P_{k} \text { s.t. (C1) } & \\
& \log _{2}(1+S(k)) \geq R_{k}^{t} \quad \forall k=1, \ldots, K
\end{aligned}
$$

where the target data rate for user $k$ is $R_{k}^{t}$. It should be noted that if $R_{k}^{t}$ is too large, the problem may become intractable. The feasibility conditions for a linear interference are given in [11]. Constraint (C5) can be rewritten in the following way:

$$
\frac{\mathcal{P}_{\mathrm{L}}^{2}(k)+2 \mathcal{P}_{\mathrm{L}}(k) \mathcal{P}_{\mathrm{LNL}}(k)+\mathcal{P}_{\mathrm{LNL}}^{2}(k)}{\mathcal{P}_{\mathrm{L}}(k) \mathcal{P}_{\mathrm{NL}}(k)+\mathcal{P}_{\mathrm{L}}(k) \mathcal{P}_{\mathrm{W}}-\mathcal{P}_{\mathrm{LNL}}^{2}(k)} \geq 2^{R_{k}^{t}}-1 \quad \forall k .
$$

As previously, we get a ratio of a posynomial over a signomial in the constraint set, that can be rewritten as a ratio of posynomials:

$$
\frac{\mathcal{P}_{\mathrm{NL}}(k)+\mathcal{P}_{\mathrm{W}}}{2^{R_{k}^{t}} \mathcal{P}_{\mathrm{L}}^{-1}(k) \mathcal{P}_{\mathrm{LNL}}^{2}(k)+\mathcal{P}_{\mathrm{L}}(k)+2 \mathcal{P}_{\mathrm{LNL}}(k)} \leq \frac{1}{2^{R_{k}^{t}-1}} \quad \forall k .
$$

We handle the posynomial ratio in the same way as before, i.e., by using the SCA with a monomial approximation of the denominator. Note that for this problem, finding a feasible initial point can be difficult. Nevertheless, we can use the solution obtained by Problem 2 in the case where $R_{k}^{t}=R_{k}$.

\section{NUMERICAL RESULTS}

A single-beam multiband satellite with an uplink in the Kaband $(27.5-29.5 \mathrm{GHz})$ is considered. The subband assignment has already been completed. The shaping filter is a square-root raised cosine filter with a roll-off of 0.25 . The HPA distortion coefficients $\omega_{1}$ and $\omega_{3}$ are 1 and 0.05 respectively. $P_{\max }=$ $50 \mathrm{~W}$ is the maximum transmit power. As in [7], the channel gains $\left\{G_{k}\right\}_{k}$ are computed using location, and we assume that one third of users are subjected to rainy weather conditions. Except otherwise stated, we have $K=6$ users.

For comparison, we also implement power allocation algorithms for nonlinearity-agnostic receiver proposed in [4], where the capacity for user $k$ used for the optimization is $\underline{C}(k)=\log _{2}\left(1+\mathcal{P}_{\mathrm{L}}(k) /\left(\mathcal{P}_{\mathrm{NL}}(k)+\mathcal{P}_{\mathrm{W}}\right)\right)$.

For each figure, we display at least the value of the considered cost function for $i$ ) the naive allocation where the users have the same transmit power $P$, which is then optimized for this considered cost function, ii) the allocation proposed by this paper, denoted by $P^{\star}$, for this considered cost function. Moreover, we plot the optimal value of the cost function for the AWGN case, i.e., when we force $\omega_{3}=0$.

In Fig. 1, we display the sum-rate versus the number of users for three different power allocation related to Problem 1. The third allocation, called exhaustive, performs a $K$-D search in the space $\left[0, P_{\max }\right]^{K}$, with a fixed number of points in the grid (namely, 10,000 points) in order to be scalable. We fix the pre-amplifier gain, denoted by $G_{\mathrm{amp}}$, at $10 \mathrm{~dB}$. This device is installed before the HPA which thus can operate in either linear or nonlinear regime, depending on how the pre-amplifier is tuned. For small value of $K$, our proposed algorithm reaches the performance of exhaustive search. But the naive and the proposed algorithm are scalable unlike the exhaustive one.

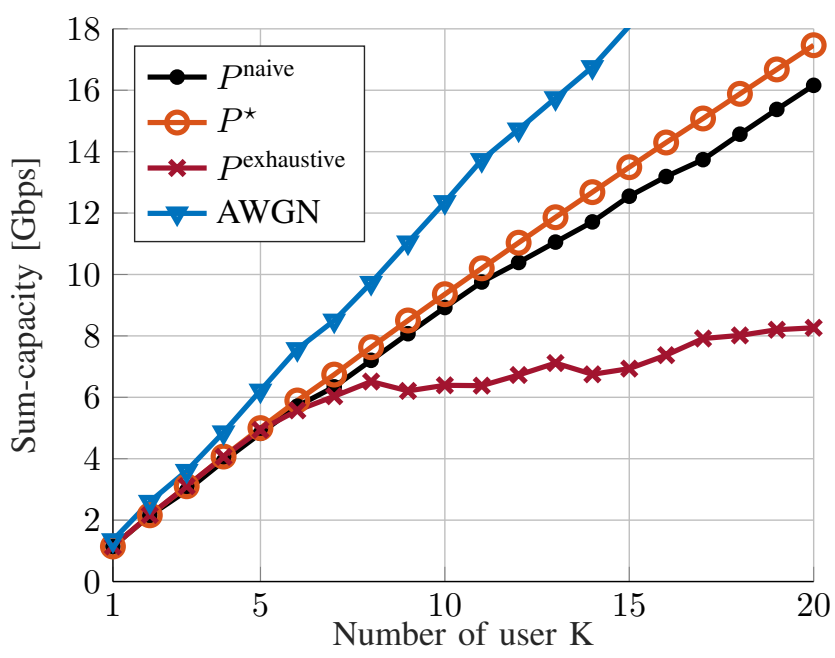

Fig. 1. Sum-capacity vs. number of users $K$ with $G_{\mathrm{amp}}=10 \mathrm{~dB}$.

In Fig. 2, we plot the sum-rate versus the pre-amplifier gain for various configurations. In the first line of the legend, the sum rate is given by $C$ and the optimization problem is Problem 1. In the second line, the sum rate is given by 
$\underline{C}$ and the optimization problem is Problem 1 where $C$ has been replaced with $\underline{C}$ (see [4]). In the third line, the sum rate is $C$ but the optimization problem has been solved with $\underline{C}$ expression. An important gain for $C$ is achieved with the proposed algorithm compared to the naive approach and the power obtained in [4].

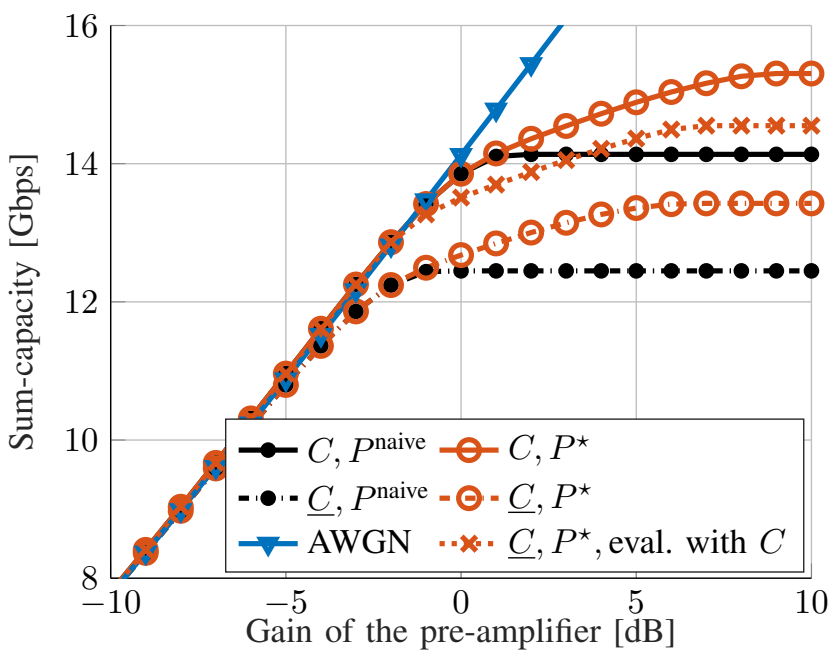

Fig. 2. Sum-capacity vs. pre-amplifier gain $G_{\mathrm{amp}}$ for nonlinearity-aware receiver optimization and nonlinearity-agnostic receiver optimization.

In Fig. 3, we display the minimum user rate versus the preamplifier gain, obtained for both power allocations related to Problem 2. We also plot the minimum user rate obtained with $\underline{C}$ when optimization has been done accordingly. Once again the proposed power allocation is for great interest.

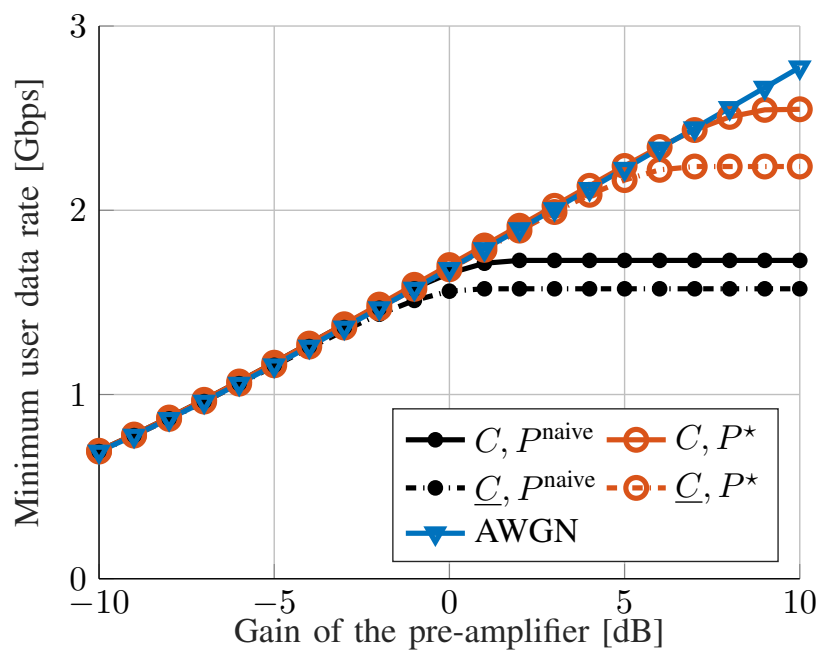

Fig. 3. Minimum user rate vs. pre-amplifier gain $G_{\mathrm{amp}}$ for nonlinearity-aware receiver optimization and nonlinearity-agnostic receiver optimization.

In Fig. 4, we plot the sum-power versus the target rate obtained for both power allocations related to Problem 3. We fix the same target for all users, and we focus on three values for the pre-amplifier gain. When the HPA operates in nonlinear regime, we notice that the proposed power allocation allows us to achieve a significantly higher target data rate.

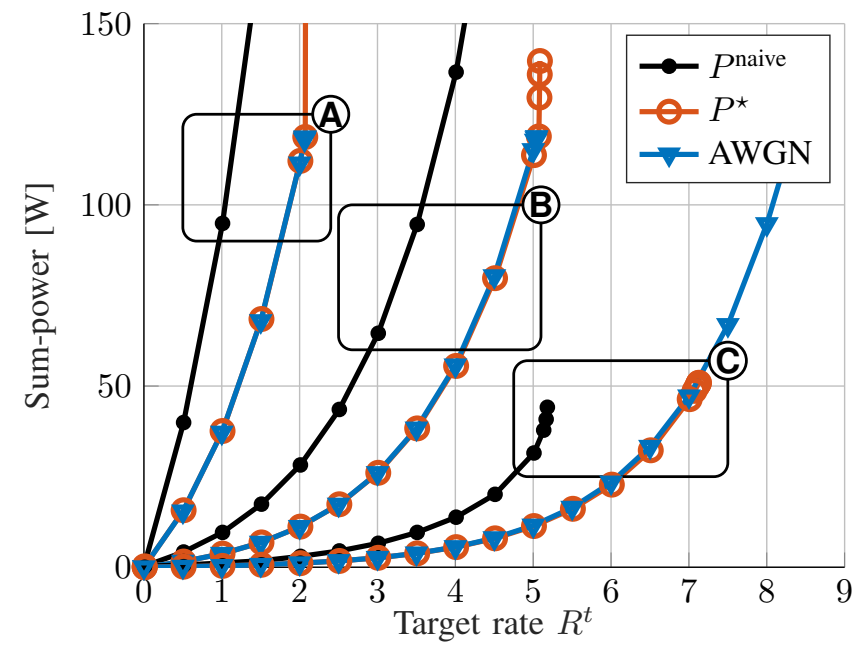

Fig. 4. Sum-power vs. target data rate $R_{k}^{t}=R^{t}$ with $G_{\mathrm{amp}} \in$ $\{-10,0,+10\} \mathrm{dB}$ (A, B and $\mathrm{C}$ respectively).

\section{CONCLUSION}

In the context of satellite communications, we proposed a scalable algorithms for power allocation when HPA operates in nonlinear regime. Signomial Programing was the relevant tool to solve the problems. For future work, we plan to exhibit closed-form expression for the capacity when multibeam satellite is considered, in order to propose related power allocation.

\section{REFERENCES}

[1] B. Evans, P. Thompson, E. Lagunas, S. K. Sharma, D. Tarchi, and V. Icolari, "Extending the usable Ka band spectrum for satellite communications: The CoRaSat project," in Wireless and Satellite Systems (WiSATS), Bradford, UK, 2015, pp. 119-132.

[2] A. Louchart, P. Ciblat, and P. de Kerret, "Resource optimization for cognitive satellite systems with incumbent terrestrial receivers," in European Signal Processing Conference (EUSIPCO), A Coruna, Spain, 2019, pp. 1-5.

[3] A. Louchart, P. Ciblat, and C. Poulliat, "Sum-capacity of uplink multiband satellite communications with nonlinear impairments," in IEEE Int. Conf. on Commun. (ICC), Montreal, Canada, 2021, pp. 1-6.

[4] - "Power allocation of uplink multiband satellite communications with nonlinear impairments," sub. to IEEE Commun. Lett.

[5] M. Chiang, C. W. Tan, D. P. Palomar, D. O'neill, and D. Julian, "Power control by geometric programming," IEEE Trans. Wireless Commun., vol. 6, no. 7, pp. 2640-2651, Jul. 2007.

[6] M. Avriel and A. C. Williams, "Complementary geometric programming," SIAM J. Appl. Math., vol. 19, no. 1, pp. 125-141, 1970.

[7] E. Lagunas, S. K. Sharma, S. Maleki, S. Chatzinotas, and B. Ottersten, "Resource allocation for cognitive satellite communications with incumbent terrestrial networks," IEEE Trans. on Cogn. Commun. and Netw., vol. 1, no. 3, pp. 305-317, Mar. 2015.

[8] S. Boyd, S.-J. Kim, L. Vandenberghe, and A. Hassibi, "A tutorial on geometric programming," Optim. Eng., vol. 8, pp. 67-127, May 2007.

[9] P. G. Kirschen and W. W. Hoburg, "The power of log transformation: A comparison of geometric and signomial programming with general nonlinear programming techniques for aircraft design optimization," AIAA/ASCE/AHS/ASC Structures, Structural Dynamics, and Materials Conf., 2018.

[10] S. Boyd and L. Vandenberghe, Convex Optimization. USA: Cambridge University Press, 2004.

[11] D. Tse and P. Viswanath, Fundamentals of Wireless Communication. USA: Cambridge University Press, 2005. 